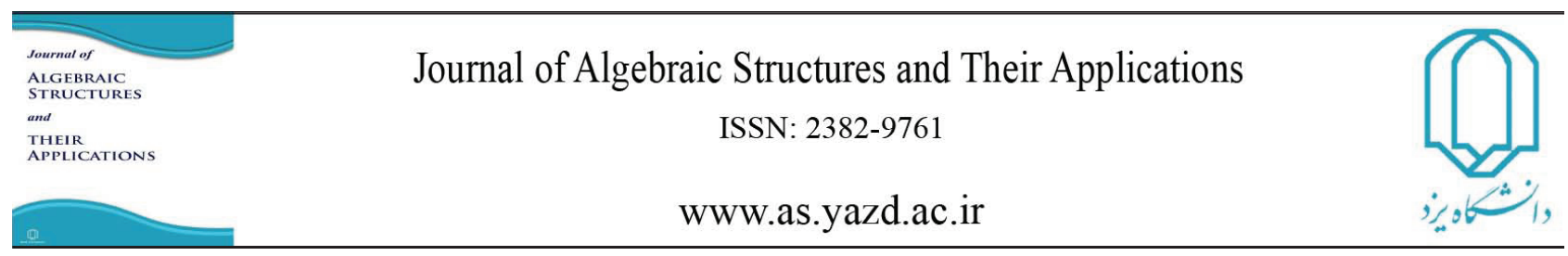

Algebraic Structures and Their Applications Vol. 5 No. 1 ( 2018 ) pp 81-88.

\title{
ON DUAL OF THE GENERALIZED SPLITTING MATROIDS
}

\author{
GH. GHAFARI*, GH. AZADI, H. AZANCHILER
}

Communicated by S. Alikhani

\begin{abstract}
Given a binary matroid $M$ and a subset $T \subseteq E(M)$, Luis A. Goddyn posed a problem that the dual of the splitting of $M$, i.e., $\left(\left(M_{T}\right)^{*}\right)$ is not always equal to the splitting of the dual of $M,\left(\left(M^{*}\right)_{T}\right)$. This persuade us to ask if we can characterize those binary matroids for which $\left(M_{T}\right)^{*}=\left(M^{*}\right)_{T}$. Santosh B. Dhotre answered this question for a two-element subset $T$. In this paper, we generalize his result for any subset $T \subseteq E(M)$ and exhibit a criterion for a binary matroid $M$ and subsets $T$ for which $\left(M_{T}\right)^{*}$ and $\left(M^{*}\right)_{T}$ are the equal. We also show that there is no subset $T \subseteq E(M)$ for which, the dual of element splitting of $M$, i.e., $\left(\left(M_{T}^{\prime}\right)^{*}\right)$ equals to the element splitting of the dual of $M,\left(\left(M^{*}\right)_{T}^{\prime}\right)$.
\end{abstract}

\section{INTRODUCTION}

Fleischner [2] defined the splitting operation on graphs as follows: Let $G$ be a connected graph and $v$ be a vertex of degree at least three in $G$. If $x=v v_{1}$ and $y=v v_{2}$ are two edges incident at $v$, then the splitting away the pair $x, y$ from $v$ results in a new gragh $G_{x, y}$ obtained from $G$ by deleting the edges $x$ and $y$, and adding a new vertex $v_{x, y}$ adjacent to $v_{1}$ and $v_{2}$. The transition from $G$ to $G_{x, y}$ is called the splitting operation on $G$.

DOI:http://dx.doi.org/10.29252/asta.5.1.81

MSC(2010): Primary: 05B35

Keywords: Binary matroid, dual of a matroid, n-connected matroid, splitting operation, cocircuit.

Received: 21 May 2018, Accepted: 19 October 2018

$*$ Corresponding author

(C) 2018 Yazd University. 
Raghunathan et al. [4] extended the notion of the splitting operation from graphs to binary matroids for every pair $x, y$ of $E(M)$. M. M. Shikare and G. Azadi [6] generalized this operation for any subset $T \subseteq E(M)$ for binary matroids as follows: Let $M$ be a binary matroid on a set $E$ and $A$ be a matrix over $G F(2)$ representing the matroid $M$. Let $T$ be a subset of $E$ and $A_{T}$ be the matrix that is obtained by adding an extra row to $A$ in which the row being zero everywhere except for the columns corresponding to $T$, where it takes the value 1 . Let $M_{T}$ be the matroid represented by the matrix $A_{T}$, we say that $M_{T}$ has been obtained from $M$ by splitting the set $T$.

Slater [7] defined the $n$-point-splitting operation on graphs as follows: Let $G$ be a graph and $u$ be a vertex of $G$ such that $\operatorname{deg}(u) \geq 2 n-2$ in which $n \in N$. Let $H$ be the graph obtained from $G$ by replacing $u$ by two adjacent vertices $u_{1}$ and $u_{2}$, if a vertex $v$ is adjacent to $u$ in $G$, written $v$ adj $u$, then make $v$ adj $u_{1}$ or adj $u_{2}$ (but not both) such that $\operatorname{deg}\left(u_{1}\right) \geq n$ and $\operatorname{deg}\left(u_{2}\right) \geq n$. We call the transition from $G$ to $H$ a $n$-point-splitting operation.

G. Azadi [6] extended the notation of $n$-point-splitting operation from graphs to binary matroid as follows. Let $M$ be a binary matroid on a set $E$ and $A$ be a matrix over $G F(2)$ representing $M$. Let $T$ be a subset of $E$, and $A_{T}^{\prime}$ be the matrix obtained by adjoining an extra row to $A$ in which the row being zero everywhere except for the columns corresponding to the elements of $T$ where it takes the value 1, and adjoining an extra column (corresponding to $a, a \notin E(M))$ with this column being zero everywhere except in the last row where it takes the value 1 . Let $M_{T}^{\prime}$ be the matroid represented by the matrix $A_{T}^{\prime}$, we say that $M_{T}^{\prime}$ has been obtained from $M$ by the element splitting of the set $T$.

Let $M$ be the matroid $(E, \mathcal{I})$ and suppose that $T \subseteq E$. Let $\mathcal{I} \mid T$ be $\{I \subseteq T: I \in \mathcal{I}\}$. Then it is easy to see that the pair $(T, \mathcal{I} \mid T)$ is a matroid. We call this matroid the restriction of $M$ to $T$, which is obtained by deleting $E-T$ from $M$. It is denoted by $M \mid T$ or $M \backslash(E-T)$. Suppose $e$ is an element of $E$ and let $M / e=\left(M^{*} \backslash e\right)^{*}$, in which $M^{*}$ is dual of $M$. We shall call $M / e$, the contraction of $M$ onto $E-\{e\}$ or the contraction of $e$ from $M$. For $X \subseteq E$, let

$$
\lambda_{M}(X)=r(X)+r(E-X)-r(M) .
$$

We call $\lambda_{M}$ the connectivity function of M. Let $k$ be a positive integer. A $k$-separation of $M$ is a partition $\{X, Y\}$ of $\mathrm{E}(\mathrm{M})$ such that $\min \{|X|,|Y|\} \geq k$, and $\lambda_{M}(X) \leq k-1$. For all $n \geq 2$, $\mathrm{M}$ is $n$-connected if, for all $k$ in $\{1,2, \ldots, n-1\}$, $\mathrm{M}$ has no $k$-separation.

Lemma 1.1. [3] Let $M$ be a matroid with ground set $E$. If $X \subseteq E$, then

$$
\lambda_{M}(X)=r(X)+r^{*}(X)-|X| .
$$

Various properties of the splitting matroids are explored in [4] and [6]. For the standard terminology in matroid we refer to [3]. 


\section{DuAl of the Binary SPlitting MATroid}

In this section, we consider the problem of finding a necessary and sufficient condition for a matroid $M$ and a subset $T$ of $E(M)$ for which $\left(M^{*}\right)_{T}=\left(M_{T}\right)^{*}$. As pointed above, the dual of the splitting of a matroid $M$ is not always equal to the splitting of it's dual. The following proposition is necessary in our discussion.

Proposition 2.1. [3] Let $A$ be a binary representation of a rank-r binary matroid $M$. Then the cocircuit space of $M$ equals to the row space of $A$. Moreover, this space has dimension $r$ and is the orthogonal subspace of the circuit space of $M$.

The following lemma is an immediate consequence of the Proposition [2.]

Lemma 2.2. Let $M$ be a binary matroid and $T \subseteq E(M)$. Then $M_{T}=M$ if and only if $T$ is a union of the disjoint cocircuits of $M$.

Corollary 2.3. Let $M$ be a binary matroid and $T \subseteq E(M)$. If $T$ is not a union of the disjoint cocircuits of $M$, then $M_{T} \neq M$ and $r^{\prime}\left(M_{T}\right)=r(M)+1$, where $r^{\prime}$ and $r$ are the rank functions of $M_{T}$ and $M$, respectively.

$$
A=\left[\begin{array}{llllll}
1 & 2 & 3 & 4 & 5 & 6 \\
1 & 0 & 0 & 1 & 0 & 1 \\
0 & 1 & 0 & 1 & 1 & 0 \\
0 & 0 & 1 & 0 & 1 & 1
\end{array}\right] \quad \text { and }
$$

(a)

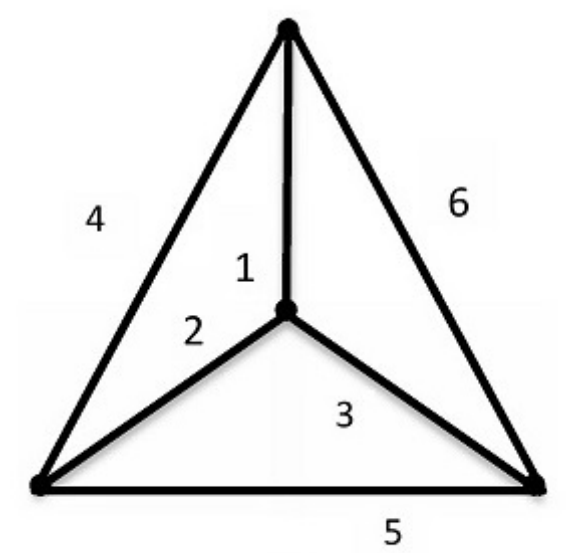

(b)

FIGURE 1

Remark 2.4. Consider the vector matroid $M$ of the matrix $A$ over $G F(2)$, that is a representation of $M\left(K_{4}\right)$ (see Figure 1), and take $T=\{3,5,6\}$. Since $T$ is a cocircuit of $M\left(K_{4}\right)$, so by Proposition [2.], $A_{T}=A$, therefore $\left(M_{T}\right)^{*}=M^{*}\left(K_{4}\right)$, (see Figure 1(a) and 2(a)).

Moreover by the following reprsentation for $\left(M^{*}\right)_{T}$ (see Figure 2(b)); We conclude that $\left(M^{*}\right)_{T}$ is different from $\left(M_{T}\right)^{*}$. 


$$
\mathrm{A}_{\top}=\left[\begin{array}{llllll}
1 & 2 & 3 & 4 & 5 & 6 \\
1 & 0 & 0 & 1 & 0 & 1 \\
0 & 1 & 0 & 1 & 1 & 0 \\
0 & 0 & 1 & 0 & 1 & 1 \\
0 & 0 & 1 & 0 & 1 & 1
\end{array}\right] \quad \text { and } \quad\left(\mathrm{M}^{*}\right)_{\top}=\left[\begin{array}{cccccc}
1 & 0 & 0 & 1 & 1 & 0 \\
0 & 1 & 0 & 0 & 1 & 1 \\
0 & 0 & 1 & 1 & 0 & 1 \\
0 & 1 & 1 & 0 & 0 & 1
\end{array}\right]
$$

(a)

(b)

Figure 2

Luis A. Goddyn asked the question: Find the condition for $M$ and $x, y$ such that the two matroids $\left(M_{x, y}\right)^{*}$ and $\left(M^{*}\right)_{x, y}$ will be the same. Santosh B. Dhotre answerd this question by the following theorem.

Theorem 2.5. [T] Let $M$ be a binary matroid, and $x, y \in E(M)$. Then $\left(M^{*}\right)_{x, y}=\left(M_{x, y}\right)^{*}$ if and only if $x$ and $y$ are in series and $\{x, y\}$ forms a circuit of $M$.

In this paper, we generalize this theorem for any subset $T \subseteq E(M)$ and exhibit a criterian for a binary matroid $M$ and subset $T$ for which $\left(M_{T}\right)^{*}$ and $\left(M^{*}\right)_{T}$ are the same.

Definition 2.6. We call a matroid $M$ an Eulerian matroid if there exist disjoint circuits $C_{1}$, $C_{2} \ldots, C_{p}$ such that $E(M)=C_{1} \cup C_{2} \cup \ldots \cup C_{p}$.

Definition 2.7. We call a matroid $M$ bipartite, if every circuit of $M$ has even cardinality.

Proposition 2.8. [8] Let $M$ be a binary matroid. Then $M$ is Eulerian if and only if $M^{*}$ is bipartite.

To prove one of our main results, we need the following theorem.

Theorem 2.9. Let $M$ be a binary matroid and $T \subseteq E(M)$. Then $\left(M^{*}\right)_{T}=\left(M_{T}\right)^{*}$ if and only if $T$ is a union of the disjoint cocircuits and also a union of the disjoint circuits of $M$.

Proof. Suppose that $T$ is a union of the disjoint cocircuits and a union of the disjoint circuits of $M$. Then by Lemma 2.2 and it's dual, $\left(M_{T}\right)^{*}=\left(M^{*}\right)_{T}$. So the result holds.

Conversely, suppose that $\left(M_{T}\right)^{*}=\left(M^{*}\right)_{T}$. We show that $T$ is a union of the disjoint cocircuits and also a union of the disjoint circuits of $M$. Suppose this is not true. We consider the following cases:

Case (i): If $T$ is neither a union of the disjoint cocircuits nor a union of the disjoint circuits of $M$. Then by Corollary $2.3, r\left(M_{T}\right)=r(M)+1$. So 


$$
\begin{aligned}
r\left(\left(M_{T}\right)^{*}\right) & =|E(M)|-(r(M)+1) \\
& =|E(M)|-r(M)-1 \\
& =r\left(M^{*}\right)-1
\end{aligned}
$$

On the other hand, by the dual of Corollary [2.3],

$$
r\left(\left(M^{*}\right)_{T}\right)=r\left(M^{*}\right)+1
$$

But, $|E(M)|-r(M)=r\left(M^{*}\right)$. Since $\left(M_{T}\right)^{*}=\left(M^{*}\right)_{T}$ so we have $r\left(M_{T}\right)^{*}=r\left(M^{*}\right)_{T}$; On the other hand by (1) and (2), we deduce that, $r\left(M^{*}\right)+1=r\left(M^{*}\right)-1$, a contradiction.

Case (ii): If $T$ is a union of the disjoint cocircuits but not a union of the disjoint circuits of $M$, then we have $\left(M_{T}\right)^{*}=M^{*}$, so

$$
\begin{aligned}
r\left(\left(M_{T}\right)^{*}\right) & =r\left(M^{*}\right) \\
& =|E(M)|-r(M) \\
& =r\left(M^{*}\right)
\end{aligned}
$$

On the other hand, by Lemma 22 along with the Corollary 22 applied to dual, we have

$$
r\left(\left(M^{*}\right)_{T}\right)=r\left(M^{*}\right)+1
$$

But $\left(M_{T}\right)^{*}=\left(M^{*}\right)_{T}$, therefore $r\left(\left(M_{T}\right)^{*}\right)=r\left(\left(M^{*}\right)_{T}\right)$. On the other hand by $(3)$ and $(4)$, we deduce that, $r\left(M^{*}\right)=r\left(M^{*}\right)+1$, a contradiction.

Case (iii): If $T$ is a union of the disjoint circuits but not the disjoint union of cocircuits of $M$, then by similar argument in case 2 on the dual of $M$, we get a contradiction, so the result holds. This completes the proof.

Corollary 2.10. Suppose $M$ is a binary matroid on $E$ and $T \subseteq E(M)$. Then $M \mid T$ is Eulerian and bipartite if and only if $\left(M^{*}\right)_{T}=\left(M_{T}\right)^{*}$.

Proof. It is an immediate cosequence of the Theorem [2.9] and the Proposition [2.8.

Corollary 2.11. Let $M$ be a n-connected binary matroid with at least $(2 n-1)$ elements. Then for every $T \subseteq E(M)$ with $|T|=n,\left(M_{T}\right)^{*} \neq\left(M^{*}\right)_{T}$.

Proof. Suppose that there is a subset $T$ with $|T|=n$ in which $\left(M_{T}\right)^{*}=\left(M^{*}\right)_{T}$. Then by Theorem [2.9, $T$ is a union of the disjoint circuits and union of the disjoint cocircuits of $M$. So 
$r(T) \leq n-k$ and $r^{*}(T) \leq n-j$ for some $j, k \geq 1$. On the other hand we have

$$
\begin{aligned}
\lambda_{M}(T) & =r(T)+r^{*}(T)-|T| \\
& =n-k+n-j-n \\
& \leq n-k-j .
\end{aligned}
$$

And since $M$ has at least $(2 n-1)$ elements, we conclude that $(T, E(M)-T)$ is a $(n-k-j+1)$ separation for $M$, a contradiction.

Definition 2.12. Let $F$ be an arbitrary field and $V=\left(v_{1}, v_{2}, \ldots, v_{n}\right)$ be a member of $V(n, F)$. The support of $V$ is $\left\{i: v_{i} \neq 0\right\}$.

Proposition 2.13. [3] Let $M$ be a binary matroid on $E$. Then $E(M)$ can be partitioned into circuits if and only if there is a basis of the cocircuit space all of whose member have even support.

Corollary 2.14. Let $M$ a binary matroid on $E$. Then $M$ is eulerian if and only if there is a matrix A representing $M$ and has even non-zero entries in each rows.

Proposition 2.15. [3] Let $C$ be a circuit of a binary matroid $M$ and $e$ be an element of $E(M)-C$. Then, in $M / e$, either $C$ is a circuit or $C$ is a disjoint union of two circuits. In both case, $M / e$ has no other circuits contained in $C$.

The following corollary is an immediate consequence of Proposition [2.15.

Corollary 2.16. 2.16 Let $M$ be binary matroid on $E, T \varsubsetneqq E$ and $y \in E-T$. If $T$ be a union of the disjoint cocircuits of $M$, then $T$ is also a union of the disjoint cocircuits of $M \mid(T \cup\{y\})$.

Theorem 2.17. Let $M$ be a binary matroid on $E$. Then for every $T \subseteq E(M)$ with $|T|=n$, $\left(M_{T}\right)^{*}=\left(M^{*}\right)_{T}$ if and only if $n=|E(M)|$ and $M$ is Eulerian and bipartite. Moreover, $n=2 k$ for some $k \in N$, that is, $n$ is even.

Proof. Since $M$ is Eulerian, bipartite and $T=E(M)$, so by Theorem [2.9, $\left(M_{T}\right)^{*}=\left(M^{*}\right)_{T}$ and the result holds.

Conversely, suppose for every $T \subseteq E(M)$ with $|T|=n,\left(M_{T}\right)^{*}=\left(M^{*}\right)_{T}$. We prove that $T=E(M)$. Suppose $T \neq E(M)$ and $y \in E(M)-T$. Since $\left(M_{T}\right)^{*}=\left(M^{*}\right)_{T}$, so by Theorem [2. $T$ is a union of the disjoint circuits and also a union of the disjoint cocircuits of $M$. Let $A$ be a matrix that representing $M$, then by Corollary [2.14, in $M[A] \mid T$ the number of non-zero entries in each row is even. Suppose that $T \cup\{y\}$ is in the first columns of $A$.

set $T^{\prime}=\left(T-\left\{x_{1}\right\}\right) \cup\{y\}$, where $x_{1} \in T$. Now $T^{\prime}$ is an $n$-element subset of $E(M)$. But $y$ must be parallel to $x_{1}$, otherwise in some row of $M[A] \mid T^{\prime}$ in which $y$ has non-zero entries, we 
have odd non-zero entries, a contradiction. Now there is an element $x_{1} \neq x_{i} \in T$ which is not parallel to $x_{1}$ and $y$, otherwise $M \mid(T \cup\{y\}) \cong U_{1,|T|+1}$, this contradicts the Corollary [2.16.

Let $T^{\prime \prime}=\{T \cup\{y\}\}-\left\{x_{i}\right\}$, where $x_{1} \neq x_{i} \in T$. Now $T^{\prime \prime}$ is an $n$-element set of $E(M)$ in which $M \mid T^{\prime \prime}$ is not Eulerian, a contradiction. So $T$ must be equal to $E(M)$. Now Theorem $[2.9$ and Proposition $[2.9$ show that $M$ is Eulerian and bipartite.

Moreover since $E(M)$ is a union of the disjoint circuits, also a union of the disjoint cocircuits of $M$, and the fact that the intersection of circuits and cocircuits of a binary matroid has even element, we conclude that $n$ is even.

\section{On Dual of the Binary ElEment SPLitTing MATroid}

In this section, we show that $\left(M^{*}\right)_{T}^{\prime} \neq\left(M_{T}^{\prime}\right)^{*}$ for every $T \subseteq E(M)$ in the element splitting. As we pointed out in abstract, in this section we prove that for every $T \subseteq E(M),\left(M^{*}\right)_{T}^{\prime} \neq$ $\left(M_{T}^{\prime}\right)^{*}$.

Lemma 3.1. [6] Let $M$ be a binary matroid on $E$. Then for any $X \subseteq E(M), r^{\prime}(X \cup\{a\})=$ $r(X)+1$ where $r$ and $r^{\prime}$ denote the rank functions of $M$ and $M_{T}^{\prime}$, respectively.

Corollary 3.2. If $M$ is a binary matroid on $E$ and $T \subseteq E(M)$, then

$$
r^{\prime}\left(M_{T}^{\prime}\right)=r(M)+1
$$

where $r$ and $r^{\prime}$ denote the rank functions of $M$ and $M_{T}^{\prime}$, respectively.

Theorem 3.3. Let $M$ be a binary matroid on $E$ and $T \subseteq E(M)$. Then for every $T \subseteq E(M)$, $\left(M^{*}\right)_{T}^{\prime} \neq\left(M_{T}^{\prime}\right)^{*}$.

Proof. Suppose that there is a $T \subseteq E(M)$, such that $\left(M^{*}\right)_{T}^{\prime}=\left(M_{T}^{\prime}\right)^{*}$. Let $r$ be the rank function. Then

$$
r\left(\left(M^{*}\right)_{T}^{\prime}\right)=r\left(\left(M_{T}^{\prime}\right)^{*}\right) .
$$

But, by Corollary [3.2, $r\left(M_{T}^{\prime}\right)=r(M)+1$, so

$$
\begin{aligned}
r\left(\left(M_{T}^{\prime}\right)^{*}\right) & =|E(M) \cup\{a\}|-(r(M)+1) \\
& =|E(M)|-r(M) \\
& =r\left(M^{*}\right) .
\end{aligned}
$$

Again by Corollary [.2,

$$
r\left(\left(M^{*}\right)_{T}^{\prime}\right)=r\left(M^{*}\right)+1
$$


Therefore by $(5),(6)$ and $(7)$ we have $r\left(M^{*}\right)+1=r\left(M^{*}\right)$, a contradiction. This completes the proof.

\section{REFERENCES}

[1] S. B. Dhotre, A note on the dual of the splitting matroid, Lobachevskii J. Math., 33, (2012), 229-231.

[2] H. Fleischner, Eulerian Graphs and Related Topics, North Holland, Amsterdam, (1990).

[3] J. G. Oxley, Matroid Theory, Oxford university press, New York, (2011).

[4] T. T. Raghunathan, M. M. Shikare and B. N. Waphare, Splitting in a binary matroid, Discrete math., 184, (1998), 267-271.

[5] M. M. Shikare, Gh. Azadi, Determination of the bases of a splitting matroid, European J. combin., 24, (2003), 45-52.

[6] M. M. Shikare, Gh. Azadi, B. N. Waphare, Generalized splitting operation and its application, J. Indian Math. Soc., 78, (2011), 145-154.

[7] P. J. Slater, A classsification of 4-connected graphs, J. Combin. Theory, 17, (1974), 281-298.

[8] D. J. A. Welsh, Eulerian and bipartite matroids, J. Comb. Theory, 6, (1969), 375-377.

\section{Gh. Ghafari}

Department of Mathematics

Urmia University, Urmia, Iran

gh.ghafari@urmia.ac.ir

Gh. Azadi

Department of Mathematics

Urmia University, Urmia, Iran

gh.azadi@urmia.ac.ir

H. Azanchiler

Department of Mathematics

Urmia University, Urmia, Iran

h.azanchiler@urmia.ac.ir 